\title{
Coherent Control of Floquet-Mode Dressed Plasmon Polaritons
}

\author{
Regine Frank \\ Institut für Theoretische Festkörperphysik, Karlsruhe Institute of Technology (KIT), \\ Wolfgang - Gaede - Strasse 1, 76131 Karlsruhe, Germany
}

(Dated: June 12, 2018)

\begin{abstract}
We study the coherent properties of plasmon polaritons optically excited on periodic nanostructures. The gold grains are coupled to a single mode photonic waveguide which exhibits a dramatically reduced transmission originating from the derived quantum interference. With a nonequilibrium description of Floquet-dressed polaritons we demonstrate the switching of light transmission through the waveguide due to sheer existence of intraband transitions in gold from right above the Fermi level driven by the external laser light.
\end{abstract}

PACS numbers: 42.50.Hz, 42.60.Rn, 42.65.Re, 42.25.Kb, 42.79.Ta

\section{INTRODUCTION}

One of the ultimate challenges of optical technologies is the realization of efficient optical switching and computing devices 3 . Therefore, quantum-optical functional elements have received increasing interest over the past years. Among the promising candidates, quantum-well structures exhibiting Kerr nonlinearities, meta-materials or plasmonic systems $\sqrt[4]{6}$ have shown the potential to be used for ultrafast optical switches $\sqrt{12}$, and expeditiously progressing ultrashort laser sciences open an avenue to their exploration $13[14$. Besides its immense technological importance, the theoretical description of externally driven non-equilibrium quantum systems is a challenge itself.

\section{SETUP}

We study a photonic silicon-on-insulator hollow core waveguide (SOI) in contact with gold nano-grains exposed to an external field. The external time-periodic field modifies the electronic band structure within the nano-grains by generating photo-induced Floquet-bands which can be attributed to the Franz - Keldysh effect 15 . All metals exhibit intraband transitions occurring within the conduction band featuring a more or less small absorption rate, whereas the governing processes are the interband transitions. In gold $(\mathrm{Au})$ a significantly different behaviour has been found and attributed to the specific electronic structure of closed packed $\mathrm{Au}$, namely to the high polarizability of the $5 d^{10}$ cores 7 . The collective resonance exhibits a large red shift to approximately $2.4 \mathrm{eV}$ which leads to the fact that the corresponding intensity is governed by the interband but its sheer existence results from the occurrence of intraband transitions which can be recognised as step like structures in the spectrum 8 . These transitions, which result from a non fermi alike distribution of states, have been observed in pump probe experiments with $\mathrm{Au}$ nano-spheres of diameters below $30 \mathrm{~nm}$. Their occurrence has been interpreted as the border from bulk like characteristics to the quantum regime of nano-particles. We show that the pump laser modifies

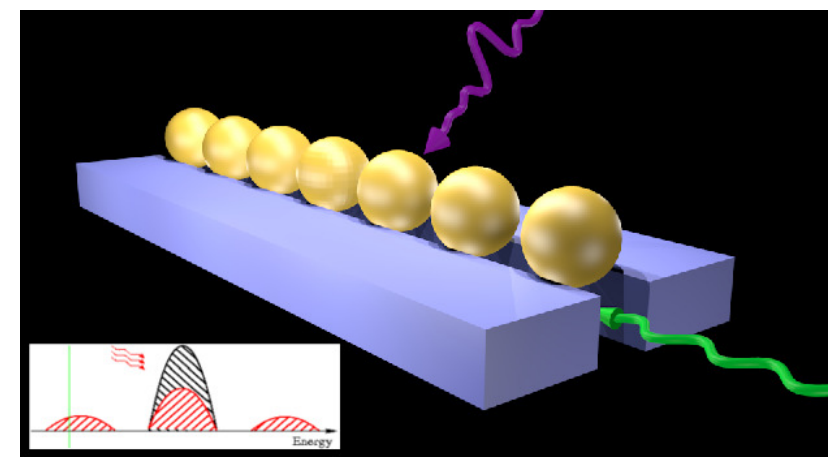

FIG. 1: (Color online) Gold nano-grains in contact with a hollow core SOI. Waveguide photons (green) and electrons in the metal form a coupled light-matter state, a polariton controlled by an external laser (pink). Inset: Sketched development of Floquet side-bands.

the single plasmon band which results in the development of Floquet-bands. Both frequency and amplitude of the external field allow easy and fast-switching control over the position in energy of these bands and sensitively control the generation of a Fano-resonance with the photonic SOI mode. We show that this switching may significantly alter the transmission properties within the SOI by the formation of polaritons, electron photon bound states, at the surface of the nano-grains. Consequentially, the potential of this quantum-optical functional element for all optical switching based on polaritonics is proven.

\section{MODEL AND METHODS}

Our setup (Fig. 1) is described by a Fröhlich Hamiltonian for fermion-boson interaction which has to be solved by applying the Keldysh formalism with respect to the non-equilibrium character of the considered processes on the femto-second time scale. We consider the SOI in contact with nano-grains, the nano-grain in the external field and finally discuss the non-equilibrium solution of the complete system in terms of electron Keldysh - Green's 
function and SOI transmission. As our starting point we choose a single-band electronic tight binding model with nearest-neighbour hopping $\frac{16}{}$, characterised by the hopping amplitude $t$, with the dispersion for a cubic lattice $\epsilon_{k}=2 t \sum_{i} \cos \left(k_{i} a\right), a$ is the lattice constant and $k_{i}$ are the components of the wave-vector. We assume a SOI supporting a single mode $\hbar \omega_{0}=2.34 \mathrm{eV}$. The SOI itself shall be coated and therefore not being exposed to laser radiation. The electrons may couple with strength $g$ weakly to SOI photons with frequency $\omega_{0}$. Hence the full Hamiltonian reads

$$
\begin{aligned}
H= & \sum_{k, \sigma} \epsilon_{k} c_{k, \sigma}^{\dagger} c_{k, \sigma}+\hbar \omega_{o} a^{\dagger} a \\
& +g \sum_{k, \sigma} c_{k, \sigma}^{\dagger} c_{k, \sigma}\left(a^{\dagger}+a\right)-t \sum_{\langle i j\rangle, \sigma} c_{i, \sigma}^{\dagger} c_{j, \sigma} \\
& +i \vec{d} \cdot \vec{E}_{0} \cos \left(\Omega_{L} \tau\right) \sum_{<i j>}\left(c_{i, \sigma}^{\dagger} c_{j, \sigma}-c_{j, \sigma}^{\dagger} c_{i, \sigma}\right) .
\end{aligned}
$$

For a clear description we treat first the setup without the external laser field, than the interaction of laser and band electrons, and finally the full compound of external laser radiation interacting with band electrons and the coupling of the latter by a Fano resonance with the waveguide mode. The Hamiltonian without the external laser field reads,

$$
H=\sum_{k, \sigma} \epsilon_{k} c_{k, \sigma}^{\dagger} c_{k, \sigma}+\hbar \omega_{o} a^{\dagger} a+g \sum_{k, \sigma} c_{k, \sigma}^{\dagger} c_{k, \sigma}\left(a^{\dagger}+a\right) .
$$

Here, we assume the spatial extension of the Au nanograins to be small $(<30 \mathrm{~nm})$ compared to the wavelength of the photonic mode inside the SOI. Therefore, the momentum of the photons is much less than the electron's momentum and we can set $q_{\text {photon }} \simeq 0$ whenever we consider the electronic subsystem. Thus, $a^{\dagger}(a)$ does not carry an index. In Eq. (1), $\epsilon_{k}$ is the electronic band energy, $c_{k, \sigma}^{\dagger}\left(c_{k, \sigma}\right)$ creates (annihilates) an electron with momentum $k$ and spin $\sigma$. $\hbar \omega_{0} a^{\dagger} a$ is the photon energy eigen-state, where $a^{\dagger}(a)$ creates (annihilates) a photon inside the SOI with energy $\hbar \omega_{0}$. The last (coupling) term on the r.h.s. is the standard term resulting from coupling the electronic and the photonic subspaces. Due to the weak interaction between the SOI photons and the electrons in the nano-grains we treat this interaction perturbatively. In second order a self-energy contribution as shown in Fig. 2 is obtained from Eq. (1). The coupling of the electronic system with a continuous energy spectrum to the photons with a discrete one leads to a Fano resonance, which is observed in the electronic density of states, as demonstrated in Fig. 3. Here we show the electron's spectral function for various frequencies of the SOI mode for coupling strength $(g / t)^{2}=0.09$ at zero temperature for a spectral width $\tau=0.005$ of the waveguide mode (measured in units of the hopping $t$ ) at half filling, yielding a suppression of the spectral function around the Fermi-level (half width $\tau$ ) where electrons are transferred to the high (low) energy tails of the spectral function. We (a)

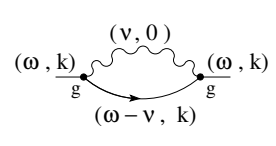

(b)

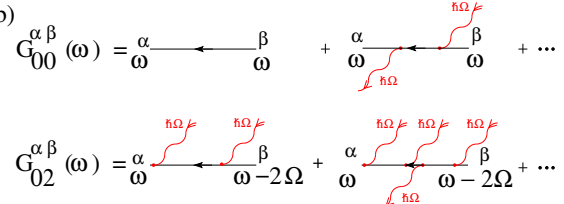

FIG. 2: (Color online)(a): Contribution to the electronic self-energy $\Sigma(\omega, k)$ in second order perturbation theory. The photon propagator does not carry a momentum as explained in the text. (b): Floquet Green's function in terms of absorption/emission of external energy quanta $\hbar \Omega_{L} . G_{00}^{\alpha \beta}(\omega), \alpha, \beta$ being Keldysh indices, e.g. represents the sum of all balanced processes. $G_{02}^{\alpha \beta}(\omega)$ describes a net-absorption of two photons.

note, that if the energy of the SOI mode $\hbar \omega_{0}$ is distinctly different from the energy $\hbar \omega$ of the band-electrons, the electronic density of states remains unchanged.

The subsystem of a nano-grain exposed to a semiclassical electromagnetic laser field is described by the Hamiltonian $(\mathrm{Lb}=$ laser + band-electrons $)$

$$
H_{L b}=-t \sum_{\langle i j\rangle, \sigma} c_{i, \sigma}^{\dagger} c_{j, \sigma}+H_{C}(\tau)
$$

where $\langle i j\rangle$ implies summation over nearest neighbores. $H_{C}(\tau)$ represents the coupling to the external, timedependent laser field, described by the electric field $\vec{E}=$ $\vec{E}_{0} \cos \left(\Omega_{L} \tau\right)$, via the electronic dipole operator $\hat{d}(\vec{x})$ with strength $\vec{d}$. It is given by

$$
H_{C}(\tau)=i \vec{d} \cdot \vec{E}_{0} \cos \left(\Omega_{L} \tau\right) \sum_{<i j>}\left(c_{i, \sigma}^{\dagger} c_{j, \sigma}-c_{j, \sigma}^{\dagger} c_{i, \sigma}\right) .
$$

The Hamiltonian specifically describes the excitation of a plasmon-polariton, which corresponds to spatially delocalized intraband electronic motion caused by an external electromagnetic wave. The accelerating energy is immediately transferred into the motion of electrons by means of single-band nearest neighbour hopping without interaction between the electrons. Due to the time dependence of the external field, Green's functions truly depend on two separate time arguments. Therefore, we use a double Fourier transform from time- to frequency space introducing relative and center-of-mass frequency

$$
\begin{aligned}
G_{m n}^{\alpha \beta}(\omega) & =\int \mathrm{d} \tau_{1}^{\alpha} \mathrm{d} \tau_{2}^{\beta} e^{-i \Omega_{L}\left(m \tau_{1}^{\alpha}-n \tau_{2}^{\beta}\right)} e^{i \omega\left(\tau_{1}^{\alpha}-\tau_{2}^{\beta}\right)} G\left(\tau_{1}^{\alpha}, \tau_{2}^{\beta}\right) \\
& \equiv G^{\alpha \beta}\left(\omega-m \Omega_{L}, \omega-n \Omega_{L}\right),
\end{aligned}
$$

where $(m, n)$ label the Floquet modes and $(\alpha, \beta)$ specify on which branch of the Keldysh contour $( \pm)$ the respective time argument resides. In general, a Floquet state is the analog to a Bloch state: The first one results from a time-periodic potential whereas the latter is the result of a space-periodic potential and both induce a band-structure. A physical interpretation of such 


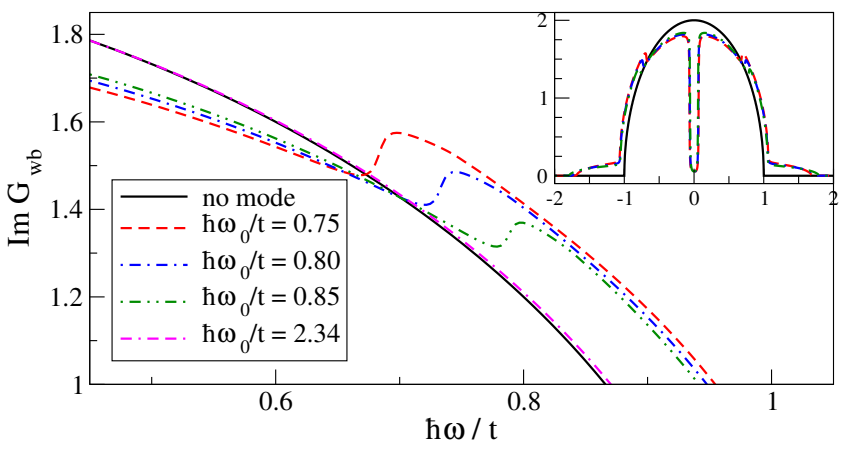

FIG. 3: (Color online) The electron's spectral function for various frequencies of the SOI mode for coupling strength $(g / t)^{2}=0.09$ (about $30 \%$ of the coupling $A_{0} / t$ ) at zero temperature for a spectral width $\tau=0.005$ of the waveguide mode. Shape and position changing of the resonance is clearly visible. If the photonic mode is energetically far off the electronic band, as for $\hbar \omega_{0}=2.34 \mathrm{eV}$, the electronic band structure remains practically unchanged. The inset shows the overall behaviour of spectral function.

a Keldysh - Floquet Green's function is given in Fig 2. The special case of non-interacting electrons allows an analytical solution for $G_{m n}(k, \omega)$ by solving the equation of motion. Including photo-induced hopping, the exact retarded Green's function for this sub-system reads

$$
G_{m n}^{R}(k, \omega)=\sum_{\rho} \frac{J_{\rho-m}\left(A_{0} \tilde{\epsilon}_{k}\right) J_{\rho-n}\left(A_{0} \tilde{\epsilon}_{k}\right)}{\omega-\rho \Omega_{L}-\epsilon_{k}+i 0^{+}}
$$

where $\tilde{\epsilon}_{k}$ represents the dispersion relation induced by the external field Eq. (3) and is different from $\epsilon$ Eq. (1). The $J_{n}$ are the cylindrical Bessel functions of integer order, $A_{0}=\vec{d} \cdot \vec{E}_{0}$ and $\Omega_{L}$ is the laser frequency. The physical Green's function is given according to

$$
G_{\mathrm{Lb}}^{R}(k, \omega)=\sum_{m, n} G_{m n}^{R}(k, \omega) .
$$

\section{RESULTS AND DISCUSSION}

We present a numerical evaluation of Eq. (6) in Fig. 4 . where $\operatorname{Im} G_{\mathrm{Lb}}^{R}(k, \omega)$, is displayed as a function of quasiparticle energy $\hbar \omega$ and external frequency $\Omega_{L}$ at zero temperature for $A_{0} / t=2.5$. As a typical value for the hopping we chose $t=1 \mathrm{eV}$. The SOI is operated at the frequency $\hbar \omega_{0}=2.34 \mathrm{eV}$ which corresponds to a frequency doubled Nd-YAG laser $\left(\hbar \omega_{0}=1.17 \mathrm{eV}\right)$. The external laser shall be characterised by $10 \mathrm{fs}$ pulses, and shall be $E_{0}=6.299 \times 10^{9} \mathrm{~V} / \mathrm{m}\left(I \sim 5.266 \times 10^{12} \mathrm{~W} / \mathrm{cm}^{2}\right)$ in the surface region of the Au grains, including Mie type field enhancement effects due to the small particle sizes. For the nano-grains we choose a damage threshold of $0.5 \mathrm{~J} / \mathrm{cm}^{2}$ and $|d|=6.528 \times 10^{-29} \mathrm{Asm}$ ( with the lattice constant for $\mathrm{Au} a_{A u}=4.08 \times 10^{-10} \mathrm{~m}$ ) resulting in

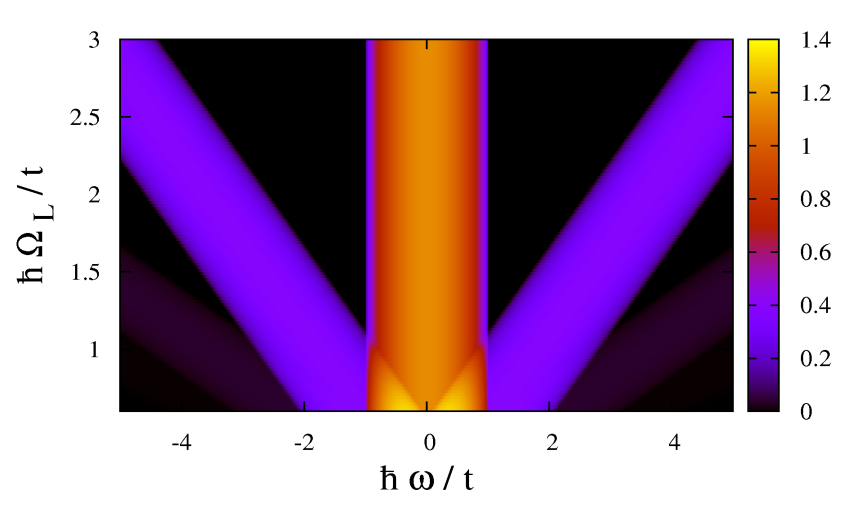

FIG. 4: (Color online) The imaginary part of the local Green's function, Eq. (6), is displayed as a function of quasiparticle energy $\hbar \omega / t$ and external frequency $\hbar \Omega_{L} / t$ at zero temperature for external amplitude $A_{0} / t=2.5$. The original semicircular DOS evolves side-bands as the laser frequency increases. Sidebands of first (bright fuchsia) and second order (faded violet) can be identified.

$A_{0} \equiv d \cdot E_{0}=2.5 \mathrm{eV}$ and the peak of the electronic response characteristics is developed after $0.3 p s$ after pulse injection, whereas the damping sets in right afterwards and so does the bleaching which is reduced with decreasing particle size ${ }^{17}$. Temperature effects are significantly lowered for nano-size particles and the observed transmission change can be totally attributed to the electronic response 18 . For later use, we also assume a density of $50 \%$ nano-grains per $10 \mu \mathrm{m}$ of the SOI. The original semicircular density of states develops photonic side-bands, the bandstructure, as the external laser frequency $\Omega_{L}$ increases. Because of the point-inversion symmetry of the underlying lattice, the first side-band represents the two-photon processes, the less pronounced second sideband the four-photon processes. Their occupation is described by the non-equilibrium distribution function as calculated from the Keldysh component of the Green's function. In a last step, we combine the relaxation processes due to the interaction between the band electrons and the SOI as described by Eq. (1), with the impinging external laser as introduced in Eq. (2). The resulting Green's function consequentially describes the SOI with $\mathrm{Au}$ nano-grains that themselves are now exposed to the external laser radiation. We treat the weak coupling between the electrons and the SOI photons by second order perturbation theory and the interaction between the electrons and external laser in terms of the Floquet theory, as demonstrated above. Since we are interested in possible switching effects, we choose as the initial situation the case where the photonic mode, $\hbar \omega_{0}=2.34 \mathrm{eV}$, is far off the electronic bandedge. Electrons arrive band energies ranging from $-1 \leq \hbar \omega / t \leq+1$. A Fano resonance is only observable in the presence of external radiation of appropriate frequency, i.e. only if the induced side-bands meet the energy of the SOI mode. This resonance will also affect the transmission properties in the SOI, since now SOI photons can be absorbed in the formation of a mixed 


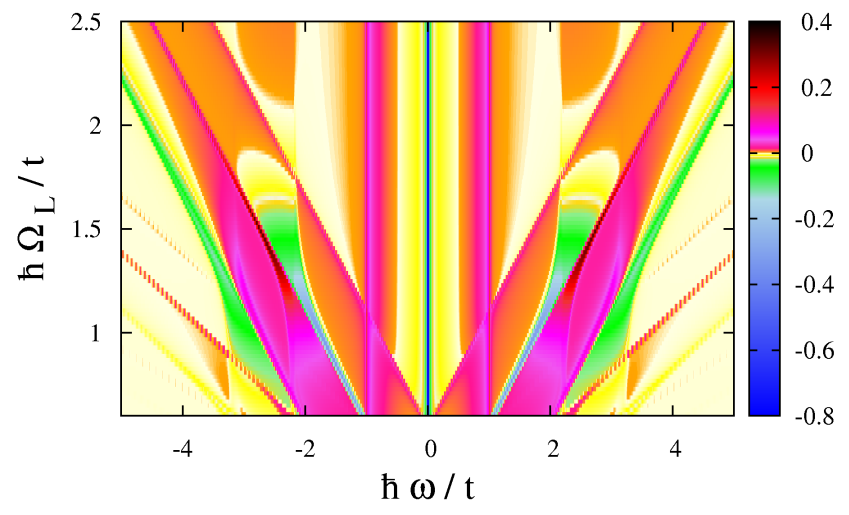

FIG. 5: (Color online) Laser induced change of the electronic density of states $\delta G\left(\omega, \Omega_{L}\right)$. A Fano resonance around quasiparticle energies $\hbar \omega_{0}=2.34 \mathrm{eV}$ is found, as soon as the external laser field redistributes the electronic spectral weight such, that the SOI mode finds electrons with about the same energy to efficiently interact with, namely to get absorbed.

state of light and matter with the laser-induced charge excitations in the Au nano-grains. Thus a waveguide polariton is created yielding to a significant reduction of the SOI's transmission. In Fig. 5, we display the laser induced change of the density of states $\delta G$ as a function of quasi-particle energy $\hbar \omega$ and external laser frequency $\Omega_{L}$. The quantity $\delta G$ measures

$$
\begin{aligned}
\delta G= & {\left[\operatorname{Im} G\left(\omega, \Omega_{L}\right)-\operatorname{Im} G_{\mathrm{Lb}}\left(\omega, \Omega_{L}\right)\right] } \\
& -\left[\operatorname{Im} G_{\mathrm{wb}}(\omega)-\operatorname{Im} G_{\mathrm{b}}(\omega)\right]
\end{aligned}
$$

the effect of the impinging laser field on the electronic density of states, and vanishes as the external laser and the coupling to the SOI is turned off. In Eq. (7), $G$ represents the Green's function including all processes,

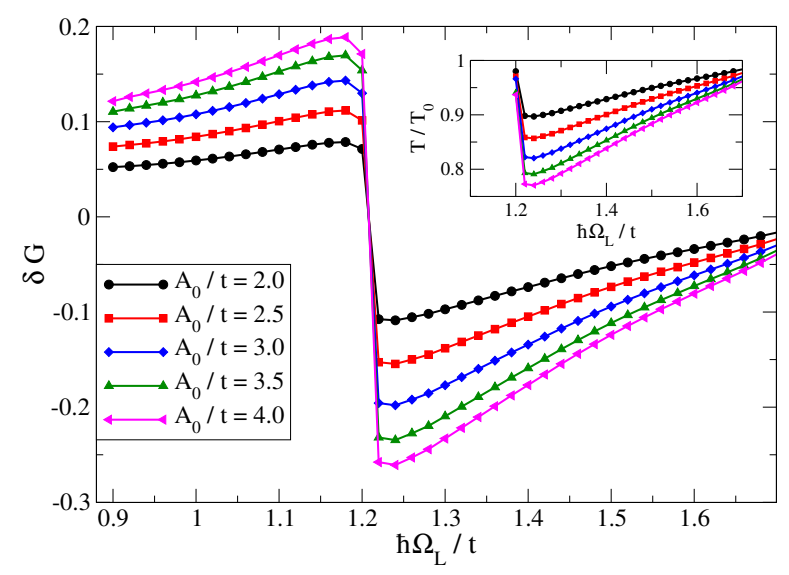

FIG. 6: (Color online) Laser induced change of the electronic DOS, $\delta G$, at fixed quasiparticle energy which meets the discrete value of the SOI mode $\hbar \omega=2.34 \mathrm{eV}=\hbar \omega_{0}$. Inset: Relative photon transmission in a SOI of unit length $l=l_{o}$ as a function of laser frequency $\Omega_{L}$.
$G_{\mathrm{Lb}}$ the interaction between the laser field and the band electrons as given in Eq. (6), $G_{\mathrm{wb}}$ describes the SOI in presence of the band electrons and is solution to Eq. (1), and finally $G_{\mathrm{b}}$ is the Green's function of just the noninteracting band electrons. In Fig. 5 the laser induced change of the electronic density of states $\delta G\left(\omega, \Omega_{L}\right)$ experiences a Fano resonance when the external laser redistributes electronic spectral weight leading to the absorption of the SOI mode at $\hbar \omega_{0}=2.34 \mathrm{eV}$. That behaviour is derived when the first photonic side-band meets the energy of the SOI mode yielding a sign change in $\delta G$ at this energy. In Fig. 6, the laser induced change of the electronic density of states $\delta G$ is shown at fixed quasiparticle energy $\hbar \omega=\hbar \omega_{0}$, where $\omega_{0}$ is the frequency of the SOI mode. Asymptotically, i.e., for large $\Omega_{L}, \delta G$ vanishes, as already indicated in Fig. 4, because in this limit there is no electronic spectral weight at the energy of the SOI mode. In the opposite limit, $\Omega_{L} \rightarrow 0$, the influence of the laser field is non-zero, because here higher-order laser induced side-bands exist, yielding spectral weight at the resonance position already for smaller laser frequencies. That result can also be concluded from the second-order side-band in Fig. 4. In a SOI of length $l$, the ratio between the initial and the transmitted intensity is given by $T \sim \exp \left(-\alpha l / l_{o}\right)$. Here $\alpha / l_{o}$, is the absorption coefficient divided by the unit length $l_{o}$, where $\alpha$ includes an average over one period of the external periodic driving field with frequency $\Omega_{L}$. We recognise that $\omega \delta G$ can be understood as the leading contribution to the relative absorption coefficient as discussed in detail in ref.19. The relative transmission of photons $T / T_{0}$ within the SOI of unit length $l=l_{o}$ is shown in Fig. 6 as a function of the external laser frequency $\Omega_{L}$. System parameters are given in caption of Fig. 5. Depending on the frequency of the driving field, an intensity drop of up to $25 \%$ is observed (Fig. 6), and by varying the length of the SOI the transmission inside the SOI can in fact be turned on and off.

\section{CONCLUSION}

We have presented a quantum field theoretical model for a SOI in contact with gold nano-grains which themselves are exposed to external laser irradiation. The strong and coherent external laser is described in terms of the Floquet theory, assuming classical behaviour of this oscillatory-in-time field, whereas the interaction with the SOI mode reflects a quantum interference. Such a description has never been proposed before to the best of our knowledge and the obtained results demonstrate the high potential of SOI polaritons for all-optical switching. Both, frequency and amplitude of the external laser control transmission through the SOI, and each of these features ensure ultrafast switching processes. 


\section{ACKNOWLEDGEMENTS}

The author thanks A. Lubatsch, K.-P. Bohnen, K. Busch, F. Hasselbach, W. Nisch, A. v. Raaij, G. Schön and M. Wegener for various fruitful discussions. P. Hom- melhoff and his group are greatfully acknowledged for highly efficient correspondence. The author is mentor of Karlsruhe School of Optics \& Photonics (KSOP) and thanks for support and funding.
1 G. Ctistis, E. Yuce, A. Hartsuiker, J. Claudon, M. Bazin, J.-M. Gérard, W. L. Vos,, Appl. Phys. Lett. 98, 161114 (2011),

2 D. Hillerkuss, R. Schmogrow, T. Schellinger, M. Jordan, M. Winter, G. Huber, T. Vallaitis, R. Bonk, P. Kleinow, F. Frey, M. Roeger, S. Koenig, A. Ludwig, A. Marculescu, J. Li, M. Hoh, M. Dreschmann, J. Meyer, S. Ben Ezra, N. Narkiss, B. Nebendahl, F. Parmigiani, P. Petropoulos, B. Resan, A. Oehler, K. Weingarten, T. Ellermeyer, J. Lutz, M. Moeller, M. Hübner, J. Becker, C. Koos, W. Freude, J. Leuthold, Nature Photonics 5, 364 (2011).

3 N.N. Negulyaev, V.S. Stepanyuk, W. Hergert, J. Kirschner, Phys. Rev. Lett. 106, 037202 (2011).

4 B. Gjonaj, J. Aulbach, P. M. Johnson, A. P. Mosk, L. Kuipers, and A. Lagendijk , Nano Letters , 12, 546550 (2012).

5 C. Stehle, H. Bender, C. Zimmermann, D. Kern, M. Fleischer, S. Slama, Nature Photonics, 5, 494 (2011).

6 A. Andryieuski, R. Malureanu, G. Biagi, T. Holmgaard, A. Lavrinenko, Optics Letters, 37, 6,1124 (2012).

7 M. M. Alvarez, J. T. Khoury, T. G. Schaaff, M. N. Shafigullin, I. Vezmar, R. L. Whetten, J. Phys. Chem. B 101, 3706 (1997).

8 M.-C. Daniel, D. Astruc, Chem. Rev. 2004, 104, 293-346 (2004).
9 B. Luk'yanchuk, N. I. Zheludev, S. A. Maier, N. J. Halas, P. Nordlander, H. Giessen, C. Tow Chong, Nature Materials, 9, 707 (2010).

10 F. B. P. Niesler, N. Feth, S. Linden, M. Wegener, Optics Letters, 36, 9, 1533 (2011).

11 N. B. Grosse, J. Heckmann, U. Woggon, Phys. Rev. Lett. 108, 136802 (2012).

12 S. Thongrattanasiri, F. H. L. Koppens,F.J. Garcia de Abajo, Phys. Rev. Lett., 108, 047401 (2012).

13 I. Park, S. Kim, J. Choi, D.-H. Lee, Y.-J. Kim, M. F. Kling, M. I. Stockman, S.- W. Kim, Nature Photonics, 5, 677 (2011).

14 M. Schenk, M. Krüger, P. Hommelhoff, Nature 475, 78 (2011), Phys. Rev. Lett. 105, 257601 (2010).

15 T. Dittrich, P. Hänggi, G.-L. Ingold, B. Kramer, G. Schön and W. Zwerger, Quantum Transport and Dissipation, Wiley-VCH, Weinheim (1998),

16 F. Pauly, J. K. Viljas, M. Bürkle, M. Dreher, P. Nielaba, J. C. Cuevas, Phys. Rev. B 84, 195420 (2011).

17 P. V. Ruijgrok, P. Zijlstra, A. L. Tchebotareva, and M. Orrit, Nano Lett. 12, 10631069 (2012).

18 G. Baffou, H. Rigneault, Phys. Rev. B 84, 035415 (2011).

19 K. Johnsen, A.-P. Jauho, Phys. Rev. B 57, 8860 (1998). 